\title{
Effects of a Launcher's External Flow on a Dual-Bell Nozzle Flow
}

\author{
Istvan Bolgar, Sven Scharnowski, and Christian J. Kähler
}

\begin{abstract}
Previous research on Dual-Bell nozzle flow always neglected the influence of the outer flow on the nozzle flow and its transition from sea level to altitude mode. Therefore, experimental measurements on a Dual-Bell nozzle with trans- and supersonic external flows about a launcher-like forebody were carried out in the Trisonic Wind Tunnel Munich with particle image velocimetry, static pressure measurements and the schlieren technique. A strongly correlated interaction exists between a transonic external flow with the nozzle flow in its sea level mode. At supersonic external flow conditions, a Prandtl-Meyer expansion about the nozzle's lip decreases the pressure in the vicinity of the nozzle exit by about $55 \%$. Therefore a new definition for the important design criterion of the nozzle pressure ratio was suggested, which considers this drastic pressure drop. Experiments during transitioning of the nozzle from sea level to altitude mode show that an interaction about the nozzle's lip causes an inherently unstable nozzle state at supersonic free-stream conditions. This instability causes the nozzle to transition and retransition, or flip-flop, between its two modes. This instability can be eliminated by designing a Dual-Bell nozzle to transition during sub-/transonic external flow conditions.
\end{abstract}

\section{Introduction}

The Ariane 5 space launcher has a geometric discontinuity, similar to a backwardfacing step (BFS), at the end of its main stage ahead of the cryogenic engine. This generates a separated shear layer from the main body, which eventually reattaches onto the nozzle of the main engine with strong local pressure fluctuations [10]. This

\footnotetext{
I. Bolgar (凶) · S. Scharnowski · C. J. Kähler

Bundeswehr University Munich, Werner-Heisenberg-Weg 39, 85577 Neubiberg, Germany e-mail: istvan.bolgar@unibw.de

(C) The Author(s) 2021

N. A. Adams et al. (eds.), Future Space-Transport-System Components under High Thermal and Mechanical Loads, Notes on Numerical Fluid Mechanics and Multidisciplinary Design 146, https://doi.org/10.1007/978-3-030-53847-7_7
} 
can lead to the aerodynamic excitement of structural modes of the main engine's nozzle, a phenomenon termed buffeting, which can cause catastrophic structural damage [17].

On a planar BFS the authors showed that the so-called 'step' and 'cross-pumping' modes are the main driving factors for the pressure fluctuations which excite buffeting [2]. With the application of passive flow control, the root mean square (RMS) of the pressure fluctuations was reduced by 35\% [3]. This load reduction is achieved through the imprinting of strong streamwise vorticity aft of the BFS with so-called 'lobes' on the step. This essentially diffuses the critical step mode and significantly weakens the cross-pumping mode. Furthermore, these lobes reduced the mean reattachment length by more than $80 \%$, thereby decreasing the moment arm of the pressure fluctuations. This decreases the integral moment of the load fluctuations by $25 \%$ [1] about the 'pivot point' at $x / h=0$. This drastic weakening of the driving factors for buffeting makes adaptive nozzle concepts, which usually are longer and heavier, a feasible option for increasing the performance of current space launchers.

A Dual-Bell nozzle, first proposed by Foster and Cowles in 1949 [7], is an adaptive nozzle which increases the thrust integral over a space launcher's trajectory. This type of nozzle is characteristic by its inflection between the throat and the exit, where the nozzle is split into two separate bells, hence a Dual-Bell. Inherently, a Dual-Bell nozzle has two operating modes; the sea level mode and the altitude mode. In the sea level mode the flow expands into first portion of the nozzle, also termed the base nozzle, where it steadily separates at the contour inflection. In this state, the nozzle flow is overexpanded, creating a low pressure jet plume and a favorable pressure gradient from the outside of the nozzle into the second portion of the nozzle termed nozzle extension. However, even at takeoff the sea level mode's overexpansion is not as extreme as it is the case for a conventional rocket nozzle. This increases the thrust integral within the troposphere while avoiding the risk of high side loads due to unsteady flow separation during the start-up of the engine. As the launcher ascends and the pressure in the atmosphere decreases below a certain threshold, the flow suddenly expands, or transitions, into the nozzle extension. This operating state is defined as the altitude mode. In the nozzle's altitude mode, the flow is expanded to a much lower pressure than would be possible with a conventional nozzle, leading to a comparatively increased thrust from the stratosphere until the main engine is finally shutdown. Stark et al. [14] recently showed how an Ariane 5 could expect a $490 \mathrm{~kg}$, or approximately $5 \%$, increase in its payload on a typical geostationary transfer orbit (GTO) mission with a change from its conventional nozzle to a Dual-Bell. Figure 1 provides a detailed overview of the geometrical and gasdynamic features of a DualBell nozzle.

The transitioning of the Dual-Bell nozzle has been the main drawback of this technology since its proposal. It generates high side-loads on the nozzle structure [8] and its mechanisms have not been fully understood. In order to keep the side loads to a minimum, the transition to altitude mode ideally has to occur instantly without re-transitioning back to sea level mode, also known as flip-flopping. Since today's conventional Bell nozzles are limited in their expansion ratio due to sideloads during engine start-up, the research on Dual-Bell nozzles has increased in the 
Fig. 1 Sketch of a Dual-Bell contour. Sea level mode is illustrated above the axis of symmetry. Altitude modes is shown below the axis of symmetry

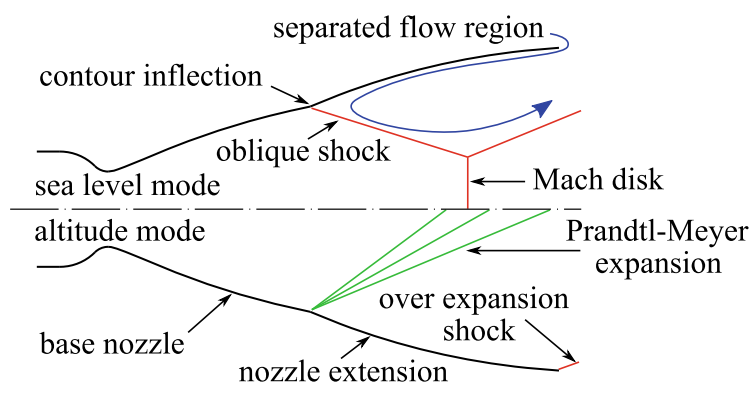

last two decades. Naturally, transition has been the major focus of many publications $[11,12,16]$ to name a few.

Some of the previously listed experiments also tried to impose external pressure fluctuations numerically or experimentally via an altitude chamber. However, the interaction of the external flow with the nozzle flow has always been neglected. However, this may have a drastic effect on the transition behavior of a Dual-Bell, since the afterbody flow of a launcher may cause large unsteady deviations in the pressure in close vicinity of the nozzle exit. Therefore, it has been the aim of the underlying research to characterize severity of these effects.

\section{Experimental Setup}

The experiments under investigation were conducted in the Trisonic Wind Tunnel Munich (TWM) at the Bundeswehr University Munich. This facility is a two-throat blow-down type wind tunnel with test section dimensions of $300 \mathrm{~mm}$ in width and $675 \mathrm{~mm}$ in height. It has an operating total pressure range of $1.2-5$ bar by which the Reynolds number can be regulated, and a Mach number range of $0.15-3.00$. During transonic measurements, the side wall suction capability of the TWM is taken advantage of. This not only helps in reducing the low momentum boundary layers on the side walls of the test section, but also reduces blockage effects at transonic conditions. The horizontal test section walls were set up with a deflection angle for all experiments, increasing the cross section in the direction of the flow by $25 \mathrm{~mm}$ over the test section length of $1.8 \mathrm{~m}$. This offsets the increasing displacement thickness of the boundary layer on the horizontal walls, thereby decreasing the pressure gradient in the test section. Table 1 provides an overview of the experimental conditions during steady-state wind tunnel runs. The \pm values in the table indicate the standard deviation of each quantity during the measurements, while the measurement uncertainty is within $\pm 1 \%$. For more details about the measurement facility the reader is referred to [2].

In order to trigger transition, the free-stream pressure in the test section is reduced over time. This is controlled via the TWM's total pressure, which causes a decrease 
Table 1 Steady free-stream flow conditions for experiments under investigation

\begin{tabular}{l|l|l|l|l}
\hline$M a_{\infty}$ & $p_{0}(\mathrm{bar})$ & $p_{\infty}($ bar $)$ & $T_{0}(\mathrm{~K})$ & $U_{\infty}(\mathrm{m} / \mathrm{s})$ \\
\hline $0.80 \pm 0.0008$ & $1.30 \pm 0.0013$ & $0.852 \pm 0.0008$ & $291 \pm 1.2$ & $\approx 258$ \\
\hline $2.00 \pm 0.0010$ & $2.50 \pm 0.0022$ & $0.320 \pm 0.0004$ & $292 \pm 1.6$ & $\approx 509$ \\
\hline
\end{tabular}

Table 2 Transient free-stream flow conditions for triggering nozzle transition

\begin{tabular}{l|l|l|l|l}
\hline$M a_{\infty}$ & $p_{0}$ (bar) & $p_{\infty}($ bar $)$ & $T_{0}(\mathrm{~K})$ & $U_{\infty}(\mathrm{m} / \mathrm{s})$ \\
\hline $0.80_{-0.01}$ & $2.0-1.4$ in $5 \mathrm{~s}$ & $1.31-0.92$ in $5 \mathrm{~s}$ & $294 \pm 0.3$ & $\approx 259$ \\
\hline $1.60_{-0.01}$ & $5.0-4.0$ in $5 \mathrm{~s}$ & $1.17-0.94$ in $5 \mathrm{~s}$ & $294 \pm 0.3$ & $\approx 447$ \\
\hline
\end{tabular}

in the static pressure at a constant Mach number, which eventually allows the nozzle to reach its transition nozzle pressure ratio $N P R_{\mathrm{tr}}$. The nozzle pressure ratio $(N P R)$ of a nozzle is defined by the ratio of the total pressure in the thrust chamber to the free-stream static pressure:

$$
N P R=\frac{p_{n, 0}}{p_{\infty}}
$$

According to Génin and Stark [11], the transition nozzle pressure ratio can be approximated by the following relation:

$$
N P R_{\mathrm{tr}}=\frac{1}{M a_{\mathrm{e}}}\left(1+\frac{\kappa-1}{2} M a_{\mathrm{e}}^{2}\right)^{\frac{\kappa}{\kappa-1}}
$$

where $M a_{\mathrm{e}}$ denotes the exit Mach number of the fully flowing nozzle extension, and $\kappa$ the ratio of specific heats for the gas at hand.

The transient wind tunnel conditions are listed in Table 2. It is important to note that the pressure was decreased linearly in the specified time frame.

\subsection{BFS Model}

The BFS model is symmetric about its horizontal plane and spans across the entire test section. It has a nose curvature which ensures subsonic conditions locally along the contour (at $M a_{\infty}=0.80$ ) [15], which then smoothly transitions into a flat plate. The model's length prior to the step is $252.5 \mathrm{~mm}$, with $102.5 \mathrm{~mm}$ of that being the flat plate. The step then has a height of $h=5 \mathrm{~mm}$ and attaches to the nozzle fairing with a length of $35 \mathrm{~mm}$. The step height to step width ratio is $1: 60$, which provides for an unaffected recirculation region due to side wall effects [6]. The overall model's thickness is $25 \mathrm{~mm}$, or $3.7 \%$ of the test section's height. At the center of the nozzle fairing with a height of $15 \mathrm{~mm}$, a 2D Dual-Bell nozzle with a nozzle exit height of 
Fig. 2 Illustration of the planar space launcher model with a 2D Dual-Bell nozzle and the measurement domains

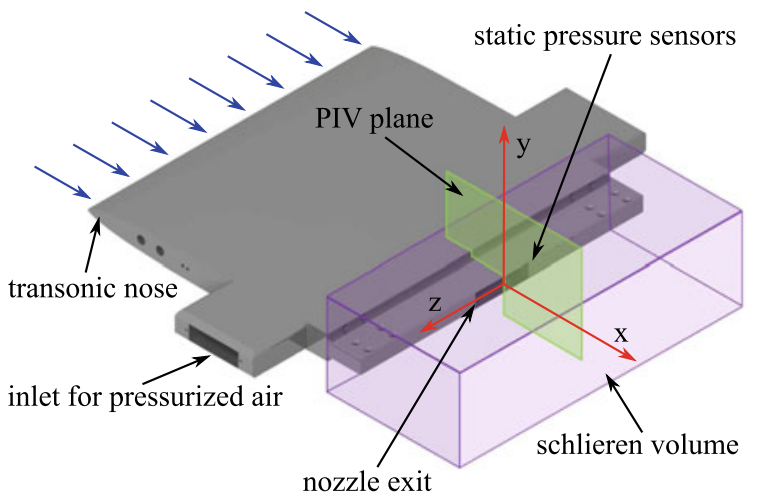

Table 3 Nozzle flow conditions of DB1 at steady state free-stream conditions

\begin{tabular}{l|l|l}
\hline$M a_{\infty}$ & $p_{n, 0}$ (bar) & $N P R$ \\
\hline 0.80 & $6.00_{-0.05}$ & $\approx 7$ \\
\hline 2.00 & $6.00_{-0.05}$ & $\approx 19$ \\
\hline
\end{tabular}

$14 \mathrm{~mm}$ spans $56 \mathrm{~mm}$ across the model. The thrust chamber is fed by two 2 " hoses, one on either side of the model (refer to Fig. 2), with the thrust chamber being symmetric about its horizontal and streamwise vertical planes.

Two different Dual-Bell contours were investigated. Both of them were designed by Chloé Génin from DLR Lampoldshausen. The reason for the two different contours is that first investigations with the initial contour showed that supersonic freestream conditions may lead to flip-flop [4]. The first contour was designed with a transition to occur at low supersonic free-stream conditions. In order to verify whether flip-flopping is indeed a supersonic artifact, a second contour was designed, which allows for transition to occur in either sub- or supersonic free-stream conditions. This work will summarize the steady-state results from the initial contour. However, the results from the second contour are used to compare the transition behavior at suband supersonic free-stream conditions.

The initial Dual-Bell contour, which will be termed DB1 for the remainder of this work, is comprised of a truncated ideal contour (TIC) base nozzle and a constant pressure nozzle extension. The nozzle throat is $2.61 \mathrm{~mm}$ in height, giving it an expansion ratio of $\epsilon=5.36$, resulting in a design exit Mach number of 3.29 in altitude mode. According to Eq. 2, this yields a transition nozzle pressure ratio of around 17. This nozzle was operated at a total pressure of about $p_{n, 0} \approx 6$ bar during the steady-state experiments. For more details refer to Table 3.

The second Dual-Bell contour under investigation, which will be termed DB2 within this work, consists of a TIC base nozzle and a overturned, or positive pressure gradient, contour nozzle extension. This kind of nozzle extension provides for a higher hysteresis between the two nozzle modes [8]. The nozzle throat is $3.26 \mathrm{~mm}$ in height, giving it an expansion ratio of $\epsilon=4.29$, resulting in a design exit Mach 
Table 4 Nozzle flow conditions of DB2 at transient free-stream conditions

\begin{tabular}{l|l|l}
\hline$M a_{\infty}$ & $p_{n, 0}$ (bar) & $N P R$ \\
\hline 0.80 & $9.8_{-0.04}^{+0.02}$ & $8.7-10.6$ \\
\hline 1.60 & $3.5_{-0.03}$ & $3.0-3.7$ \\
\hline
\end{tabular}

number of 2.73 in altitude mode. According to Eq. 2, this yields a transition nozzle pressure ratio of around 9. This nozzle was operated at a total pressure of about $p_{n, 0} \approx$ 9.8 bar at transonic transient free-stream conditions, and at about $p_{n, 0} \approx 3.5$ bar at supersonic transient free-stream conditions. For more details refer to Table 4.

\subsection{Measurement Techniques}

Particle image velocimetry (PIV) was used to capture instantaneous flow fields in a streamwise vertical field of view (FOV) for the steady-state experiments with DB1. For each test case, 500 double images with a statistically independent frequency of $15 \mathrm{~Hz}$ were recorded. A final vector grid spacing of $285 \mu \mathrm{m}$ was obtained after processing. Averaged flow field data was obtained by ensemble averaging the instantaneous vector fields. PIV was accompanied by static pressure measurements on the nozzle fairing just ahead of the nozzle lip, and on the base surface next to the nozzle exit when turning about the nozzle lip. Three sensors were placed on each surface, recording at $200 \mathrm{~Hz}$. Since the pressure values on each surface were comparable between the three sensors, the average data of the three sensors on each surface is provided within this work. For more details about the PIV setup, the reader is referred to [4].

The transitioning of the nozzle during the transient experiments with DB2 was captured with the schlieren technique. The experiments summarized in this work used a single-color schlieren system, which allows the visualization of density gradients, isentropic compression and expansion waves, and compressible shear layers. The light was focused onto a high-speed camera sensor recording at $10 \mathrm{kHz}$. For a detailed description of the schlieren system installed at the TWM facility, the reader is referred to $[5,9]$. The nozzle condition was then quantified by correlating each recorded schlieren image to a sample schlieren image with a known nozzle state. A normalized correlation value close to 1 signifies that the evaluated schlieren image is in the same nozzle mode as the sample image. On the other hand, a normalized correlation value close to 0 shows that the evaluated schlieren image is in the opposite nozzle mode as the sample image. 


\section{Results}

\subsection{Steady-State Sea Level Mode}

The steady-state sea level mode with DB1 can be obtained at transonic free-stream conditions at $M a_{\infty}=0.80$. This can clearly be seen in Fig. 3, where a reverse flow region develops in the nozzle extension. In this averaged flow field, a streamline from the outer flow extends into the nozzle, indicating that an interaction may be present. For a detailed analysis of the interaction based on instantaneous vector field data, the reader is referred to [5]. One should also note the provided static pressure values in this figure. The free-stream pressure about the step reduces by about $7 \%$ on the nozzle fairing, just ahead of the nozzle lip. About the nozzle lip, the static pressure reduces by another $2 \%$, resulting in an overall pressure decrease of about $9 \%$ from the free-stream to the pressure in close vicinity of the nozzle. This pressure decrease is not accounted for in the classically defined NPR in Eq. 1, however it will have an effect on it and thus transition.
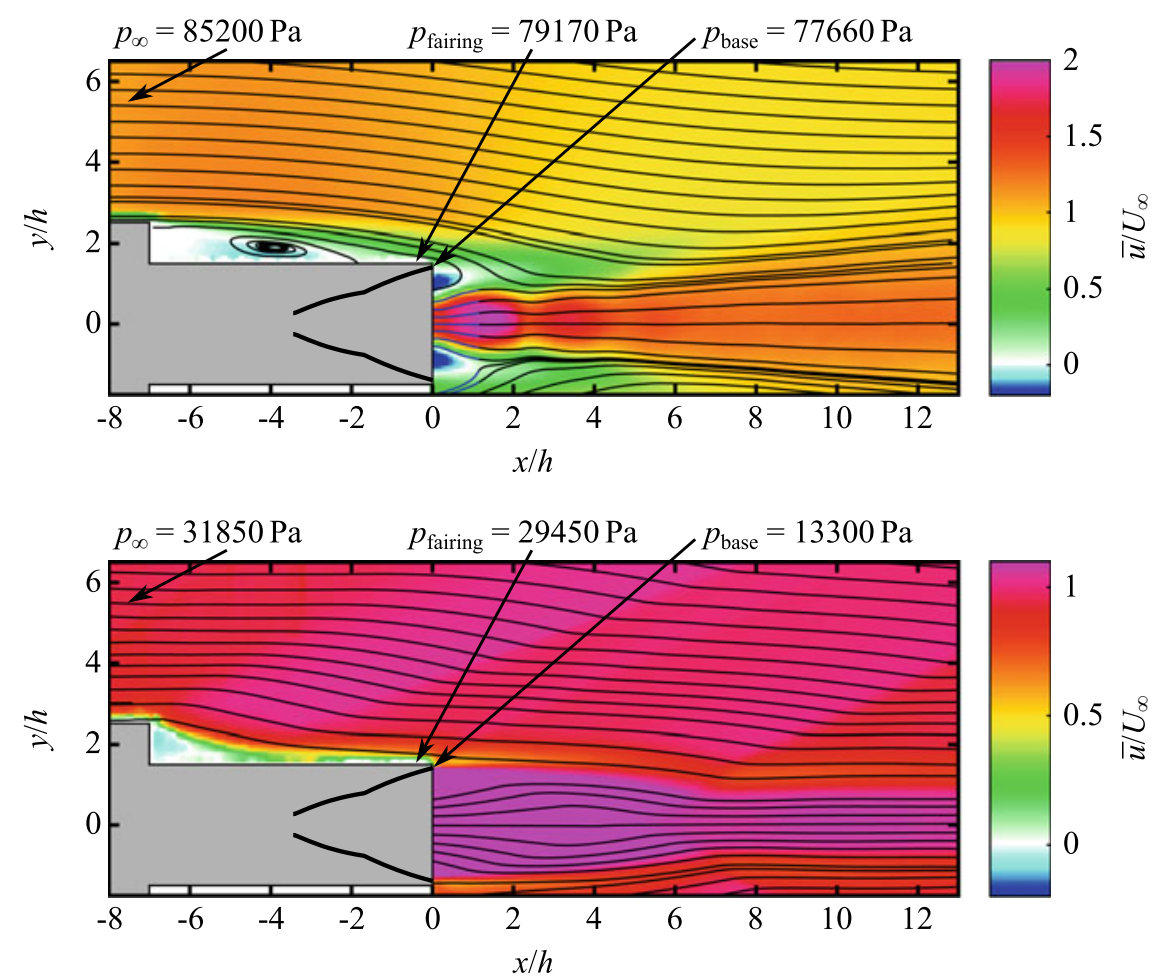

Fig. 3 Averaged streamwise component of the velocity vector in the streamwise vertical FOV for DB1. Top: Sea level mode at $M a_{\infty}=0.80$ and $N P R \approx 7$. Bottom: Altitude mode at $M a_{\infty}=2.00$ and $N P R \approx 19$ 


\subsection{Steady-State Altitude Mode}

With the DB1 contour, altitude mode could only be obtained in supersonic external flow conditions. In Fig. 3, the nozzle flow has fully transitioned into the nozzle extension. During these supersonic steady-state conditions the flow expands about the nozzle lip via a Prandtl-Meyer expansion. Prandtl-Meyer expansions are characterized by drastic pressure reductions, as can be inferred by the provided static pressure data in this figure.

Similar to the transonic case, the free-stream pressure reduces by about $7.5 \%$ about the BFS to the end of the nozzle fairing. However, about the nozzle lip, the pressure decreases by nearly $55 \%$, resulting in an overall pressure reduction of around $58 \%$ in close vicinity of the nozzle exit! This pressure reduction is bound to have an effect on the transitioning of the nozzle, thus an effective nozzle pressure ratio ( $\left.N P R_{\mathrm{eff}}\right)$, which accounts for the large pressure drop about the nozzle lip, has been defined [4]. This will be elaborated on in the Sect.3.3.

\subsection{Transition}

For the investigations on transitioning, the DB2 contour was analyzed with schlieren recordings. In Fig. 4, two snap shots each, separated by $6 \mathrm{~ms}$, are provided for the transition event in trans- and supersonic external conditions. For reference, the complete transition process takes around $1 \mathrm{~ms}$, which was processed by analyzing the individual schlieren recordings.

The red square in the images is the interrogation window in which the nozzle mode is evaluated in (refer to Sect. 2.2 for the method). The scalar result of this processing method can be normalized, thus yielding values between 0 to 1 . This number can be quantified as the nozzle mode criterion (NMC), which gives reliable information about the instantaneous nozzle mode. Figure 5 plots the NMC across each schlieren image, or in other words versus time.

In transonic free-stream conditions at the top of Fig. 5, transition occurs around $N P R \approx 8.6$ as $N P R$ increases constantly. This is a desired kind of transition, since it occurs quickly and only once. By decreasing $N P R$, it was verified that retransition occurs at $N P R \approx 8.3$, indicating that this nozzle has a hysteresis as intended by its nozzle extension's contour. Hysteresis can be quantified as follows [11]:

$$
H=\frac{N P R_{\mathrm{tr}}-N P R_{\mathrm{retr}}}{N P R_{\mathrm{tr}}} \times 100 \%
$$

where $N P R_{\text {retr }}$ is the retransition $N P R$. This yields a hysteresis value of around $3.5 \%$ for DB2.

With a supersonic external flow, transitions are followed by retransitions over a wide range of $N P R$ as can be seen at the bottom of Fig. 5. This is also known as 

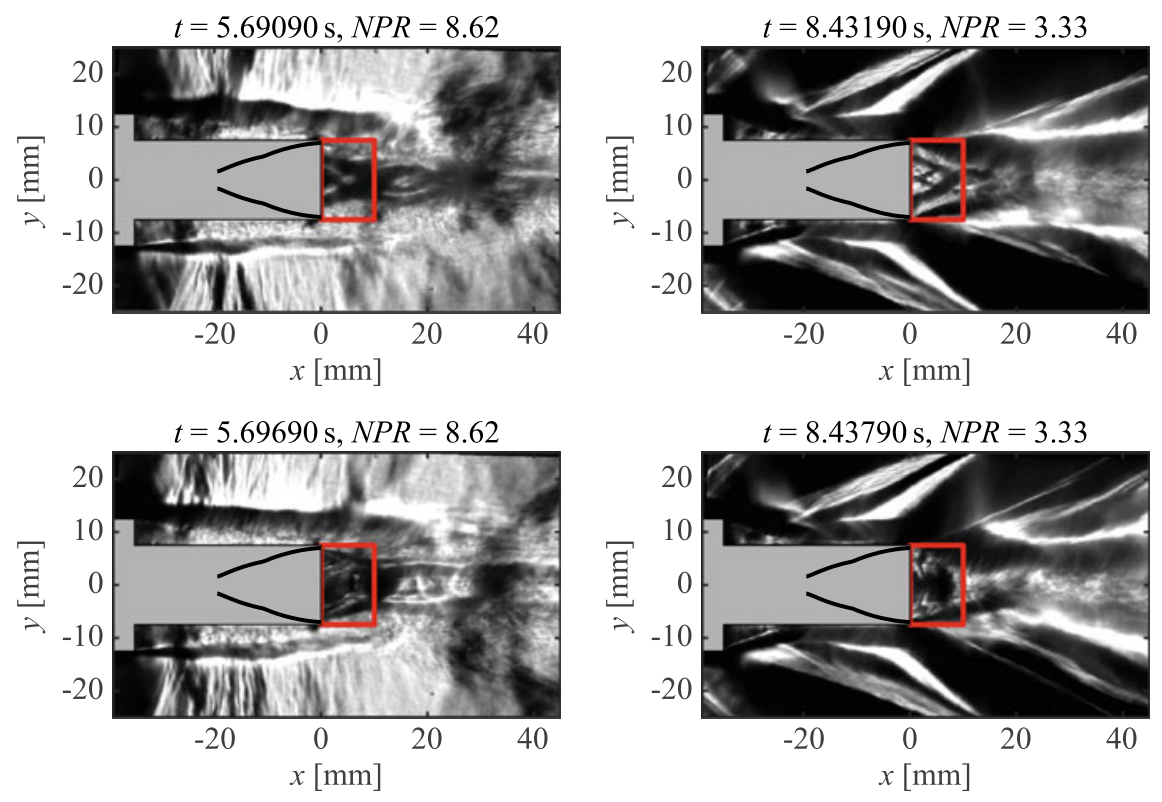

Fig. 4 Schlieren recordings of transitioning of DB2 from sea level to altitude mode (top to bottom). Left: transition at $M a_{\infty}=0.80$. Right: transition at $M a_{\infty}=2.00$
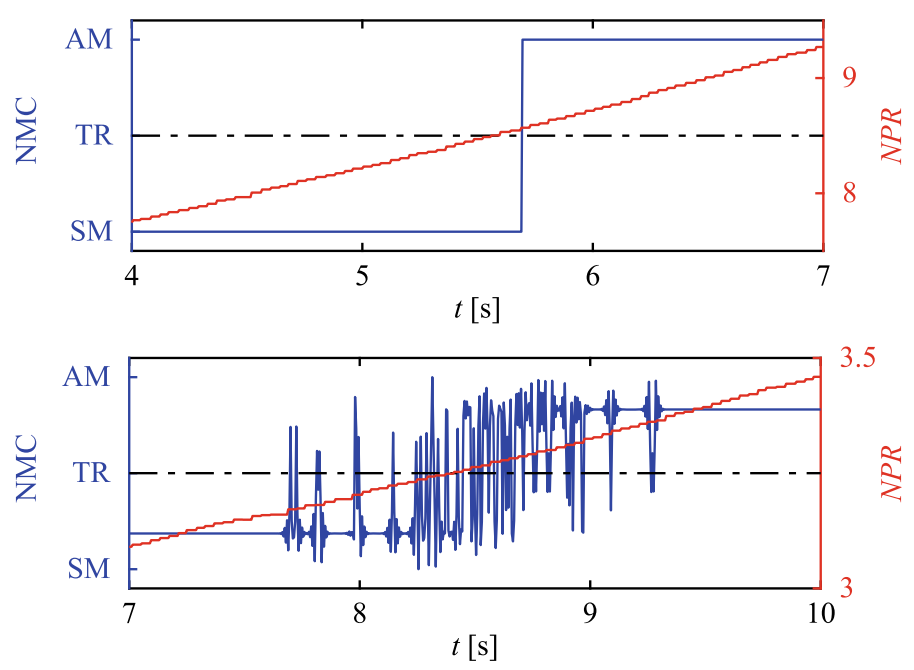

Fig. 5 NMC versus time. Top: Transition at transonic conditions. Bottom: Transition at supersonic conditions 


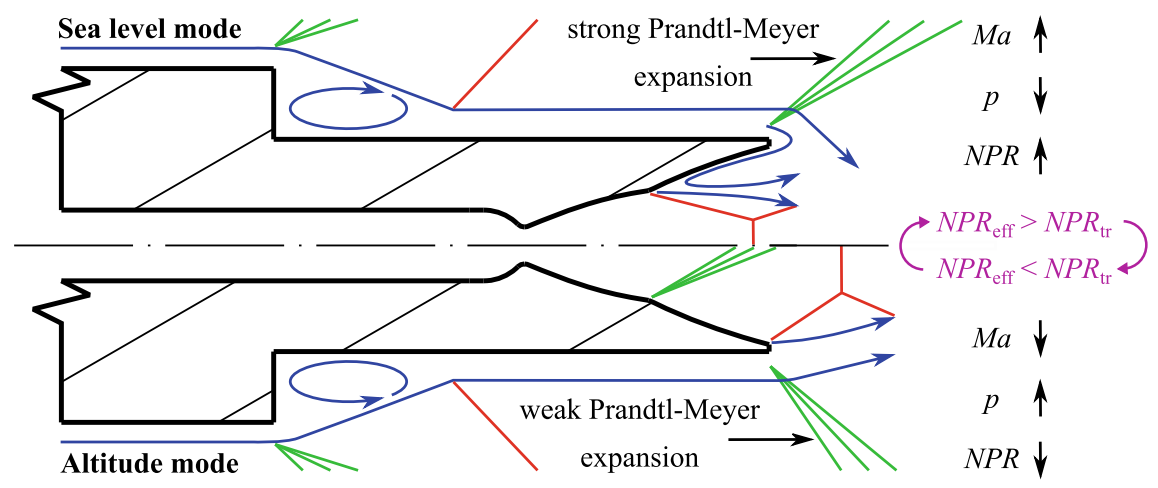

Fig. 6 Destabilizing mechanism of a Dual-Bell nozzle causing flip-flop in supersonic external flow

flip-flopping, which is apparently excited by the presence of a supersonic external flow. Also notice that transition occurs at $N P R_{\mathrm{tr}} \approx 3.25 \pm 0.15$, whereas the design $N P R_{\mathrm{tr}}$ is around 9. This is due to the Prandtl-Meyer expansion about the nozzle lip as described in the previous section. Similar to the transition itself, the retransition also takes about $1 \mathrm{~ms}$, which would translate to a period of $2 \mathrm{~ms}$ for one flip-flop event. If this were to occur consistently, the flip-flop would have a frequency of around $500 \mathrm{~Hz}$. In the time frame from about $7.8 \mathrm{~s}-9.3 \mathrm{~s}, 29$ transitions occur between $N P R=3.1$ - 3.4. This indicates that even though DB2 was designed with an overturned nozzle extension, a supersonic external flow has a larger destabilizing effect on the jet plume than the stabilizing hysteresis of this contour. A model by which the nozzle flow is destabilized has been provided by Bolgar et al. [5], illustrated in Fig. 6.

Essentially, when the Dual-Bell is in sea level mode and the external static pressure decreases due to the flow acceleration about the nozzle lip, $N P R_{\text {eff }}$ increases above $N P R_{\text {tr }}$, resulting in transition. When the jet plume reaches altitude mode, the external flow is displaced, which reduces its acceleration and expansion about the lip. In turn, $N P R_{\text {eff }}$ decreases below $N P R_{\text {tr }}$ which leads to retransition. Within a certain range of $N P R$, the jet plume is unstable in either condition.

Even though this model was created using the insights from the supersonic experiments, the destabilizing effect after transition also must exist below sonic external conditions, however the hysteresis of the nozzle is larger than the destabilization in that regime. Section 3.2 outlined how $N P R_{\text {eff }}$ is drastically effected by a supersonic external flow. Regardless of sub- or supersonic external flow conditions, $N P R_{\text {eff }}$ for a Dual-Bell nozzle should also consider the pressure drop within the recirculation region extending into the nozzle extension when the nozzle is in its sea level mode. Thus, for a Dual-Bell nozzle two separate definitions of the effective nozzle pressure ratio need to be defined as follows: 


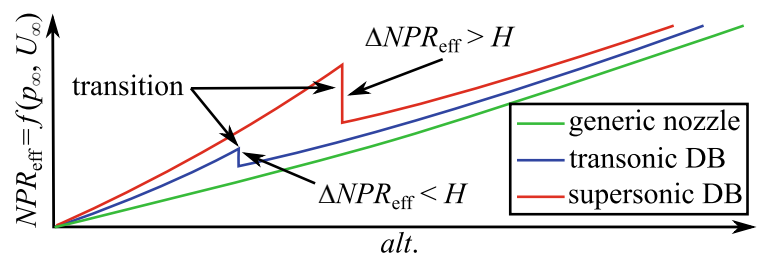

Fig. $7 N P R$ versus alt. Notice that the magnitude of $\triangle N P R_{\text {eff }}$ in comparison to $H$ dictates whether the jet plume is stable or unstable. The curves are parabolic due to the effect of the increase in velocity versus altitude (alt.)

Sea level mode $\left(N P R_{\mathrm{eff}}<N P R_{\mathrm{tr}}\right): \quad N P R_{\mathrm{eff}}=\frac{p_{n, 0}}{p_{\text {wall, extension }}}$

$$
\text { Altitude mode }\left(N P R_{\mathrm{eff}}>N P R_{\mathrm{tr}}\right): \quad N P R_{\mathrm{eff}}=\frac{p_{n, 0}}{p_{\text {lip, base }}}
$$

where $p_{\text {wall, extension }}$ is the static pressure along the wall contour in the nozzle extension after the inflection and $p_{\text {lip, base }}$ is the static pressure on the base surface of the nozzle lip. From this equation one can see that $N P R_{\text {eff }}$ is not a continuous function as is the case for a launcher with a generic nozzle ascending through the atmosphere. Rather, as the Dual-Bell nozzle flow transitions, a discontinuity in N P R occurs over a launcher's trajectory. Depending on the direction of the discontinuity, this could either stabilize the transition process by increasing $N P R_{\text {eff }}$ directly after transitioning, or destabilize the transition process if $N P R_{\text {eff }}$ decreases after the transitioning.

As a launcher increases its velocity during sea level mode, the reverse flow velocities within the nozzle extension will increase. This causes the static pressure to decrease, increasing $N P R_{\text {eff }}$ as a function of the vehicle's velocity. This condition is conducive for an earlier than predicted transition process. As soon as the nozzle flow transitions, the reverse flow region in the extension disappears, increasing the pressure in the external flow surrounding the jet plume. This causes $N P R_{\text {eff }}$ to drop, making the transition process of a Dual-Bell nozzle inherently unstable during flight conditions of a launcher! If the discontinuity in $N P R_{\text {eff }}$ is larger than the hysteresis $H$, then the instability condition is satisfied, meaning that the jet plume will flipflop (refer to Fig. 7). Future experiments should verify the validity of the Dual-Bell stability model provided in Fig. 7.

\section{Summary and Conclusions}

In summary, when a space vehicle is in motion, $N P R$ is not only a function of altitude, but also of velocity, since the pressure in the external flow surrounding the jet plume changes. Additionally for a Dual-Bell nozzle $N P R_{\text {eff }}$ has a step function at 
transition, which naturally destabilizes either mode in which the nozzle is operating in. This destabilization is further amplified in supersonic external flow conditions, which excites flip-flopping of the jet plume between its two modes. Below sonic external flow conditions, an overturned nozzle extension has a sufficient hysteresis between the transitioning and the retransitioning nozzle pressure ratio to counteract the destabilization of the transition itself. Thus, in sub- or transonic flow conditions, a natural transition of the Dual-Bell nozzle flow is possible, which was verified by the underlying experiments. In supersonic flow, a shortened nozzle fairing length would in theory also weaken the supersonic amplification of the destabilization. Future experiments should investigate, whether a shorter nozzle fairing length can reduce or eliminate the supersonic interaction about the nozzle lip. However, when considering the trajectory performance of a launcher, a Dual-Bell nozzle designed to transition in transonic flight may be the ideal solution, where a natural transition can occur in a stable manner.

\section{References}

1. Bolgar, I.: On the performance increase of future space launchers: investigations of buffeting, its reduction via passive flow control, and the Dual-Bell nozzle concept at trans- and supersonic flight conditions. Doctoral dissertation, Bundeswehr University Munich, Neubiberg, Germany (2019)

2. Bolgar, I., Scharnowski, S., Kähler, C.J.: The effect of the Mach number on a turbulent backward-facing step flow. Flow Turbul. Combust. 101(3), 653-680 (2018). https://doi.org/ 10.1007/s10494-018-9921-7

3. Bolgar, I., Scharnowski, S., Kähler, C.J.: Passive flow control for reduced load dynamics aft of a backward-facing step. AIAA J. 57(1), 120-131 (2019). https://doi.org/10.2514/1.J057274

4. Bolgar, I., Scharnowski, S., Kähler, C.J.: Experimental analysis of the interaction between a dual-bell nozzle with an external flow field aft of a backward-facing step. In: New Results in Numerical and Experimental Fluid Mechanics. Series: Notes on Numerical Fluid Mechanics and Multidisciplinary Design, vol. XII, pp. 405-415. Springer Nature Switzerland AG, Cham (2019). https://doi.org/10.1007/978-3-030-25253-3_39

5. Bolgar, I., Scharnowski, S., Kähler, C.J.: In-flight transition of a Dual-Bell nozzle - transonic vs. supersonic transition. In: Proceedings of the 8th European Conference for Aeronautics and AeroSpace Sciences (EUCASS), Madrid, Spain (2019). https://doi.org/10.13009/ EUCASS2019-670

6. de Brederode, V.A.: Three-dimensional effects in nominally two-dimensional flows. Doctoral dissertation, Imperial College London, London, Great Britain (1975)

7. Foster, C., Cowles, F.: Experimental study of gas-flow separation in overexpanded exhaust nozzles for rocket motors. JPL Progress Report, 4-103, Jet Propulsion Laboratory, California Institute of Technology, Pasadena, CA, USA (1949)

8. Frey, M., Hagemann, G.: Critical assessment of dual-bell nozzles. J. Propuls. Power 15(1), 137-143 (1999). https://doi.org/10.2514/2.5402

9. Hampel, A.: Auslegung, Optimierung und Erprobung eines vollautomatisch arbeitenden Transsonik-Windkanals. Doctoral dissertation, Bundeswehr University Munich, Neubiberg, Germany (1984)

10. Hannemann, K., Lüdeke, H., Pallegoix, J.-F., Ollivier, A., Lambaré, H., Maseland, J.E.J., Geurts, E.G.M., Frey, M., Deck, S., Schrijer, F.F.J., Scarano, F., Schwane, R.: Launch vehicle base buffeting - recent experimental and numerical investigations. In: Proceedings 7th European Symposium on Aerothermodynamics for Space Vehicles, Brugge, Belgium (2011) 
11. Nürnberger-Génin, C., Stark, R.H.: Experimental study on flow transition in dual bell nozzles. Shock Waves 126(3), 497-502 (2010). https://doi.org/10.2514/1.47282

12. Pergio, D., Schwane, R., Wong, H.: A Numerical comparison of the flow in conventional and dual bell nozzles in the presence of an unsteady external pressure environment. In: Proceedings of the 39th Join Propulsion Conference and Exhibit, Huntsville, AL, USA (2003). https://doi. org/10.2514/6.2003-4731

13. Rodgers, J.L., Nicewander, W.A.: Thirteen ways to look at the correlation coefficient. Am. Stat. 42(1), 59-66 (1988). https://doi.org/10.1080/00031305.1988.10475524

14. Stark, R.H., Génin, C., Schneider, D., Fromm, C.: Ariane 5 performance optimization using dual-bell nozzle extension. J. Spacecr. Rocket. 53(4), 743-750 (2016). https://doi.org/10.2514/ 1.A33363

15. Statnikov, V., Roidl, B., Meinke, M., Schröder, W.: Analysis of spatio-temporal wake modes of space launchers at transonic flow. In: Proceedings of 54th AIAA Aerospace Sciences Meeting, San Diego, CA, USA (2016). https://doi.org/10.2514/6.2016-1116

16. Verma, S.B., Stark, R.H., Haidn, O.: Effect of ambient pressure fluctuations on dual-bell transition behavior. J. Propuls. Power 30(5), 1192-1198 (2014). https://doi.org/10.2514/1.B35067

17. Winterfeldt, L., Laumert, B., Tano, R., James, P., Geneau, F., Blasi, R., Hagemann, G.: Redesign of the vulcain 2 nozzle extension. In: Proceedings of 41st AIAA/ASME/SAE/ASEE Joint Propulsion Conference \& Exhibit, Tucson, Arizona, USA (2005). AIAA 2005-4536

Open Access This chapter is licensed under the terms of the Creative Commons Attribution 4.0 International License (http://creativecommons.org/licenses/by/4.0/), which permits use, sharing, adaptation, distribution and reproduction in any medium or format, as long as you give appropriate credit to the original author(s) and the source, provide a link to the Creative Commons license and indicate if changes were made.

The images or other third party material in this chapter are included in the chapter's Creative Commons license, unless indicated otherwise in a credit line to the material. If material is not included in the chapter's Creative Commons license and your intended use is not permitted by statutory regulation or exceeds the permitted use, you will need to obtain permission directly from the copyright holder.

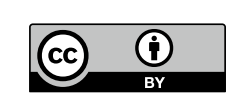

